\title{
Community Detection in Social Networks Using Information Diffusion
}

\author{
Alireza Hajibagheri, Hamidreza Alvari, Ali Hamzeh, Sattar Hashemi \\ Computer Science and Engineering Department \\ Shiraz University \\ Shiraz, Iran \\ \{hajibagheri, alvari, ali\}@cse.shirazu.ac.ir, s_hashemi@shirazu.ac.ir
}

\begin{abstract}
Discovering communities in popular social networks like Facebook has been receiving significant attentions recently. In this paper, inspired from real life, we have addressed the community detection problem by a framework based on Information Diffusion Model and Game Theory. In this approach, we consider each node of the social network as a selfish agent which has interactions with its neighbors and tries to maximize its total utility (i.e. received information). Finally community structure of the graph reveals after reaching to the local Nash equilibrium of the game. Experimental results on the benchmark social media datasets, synthetic and real world graphs demonstrate that our method is superior compared with the other state-of-the-art methods.
\end{abstract}

Keywords-social network; game theory; information diffusion; nash equilibrium; community structure.

\section{INTRODUCTION}

There are several intriguing applications which motivate the researchers from different disciplines to conduct an analysis on social networks. The aforementioned applications include finding related topics within World Wide Web [1], friend suggestion in social networks like Facebook [2] and discovering functional groupings in metabolic networks [3] and etc. As a complex graph-based structure, a social network contains nodes connecting by one or more specific types of interdependency such as friendship, common interest, financial exchange, etc.

Among the huge number of interesting properties of social networks we can mention the well-known property community structure [4]. The problem of community detection has been devoted a massive research compared with the other issues related to social network analysis. Put it simply, this problem is defined as clustering the vertices of a given underlying graph of the network into communities or groups based on some predefined measures. However, this problem, intuitive at first sight, is not well defined because its main components (e.g. the community concept) are not precisely defined and there are often some ambiguities in their definitions. This has resulted in presenting so many interpretations in the literature (See [5] and [6] for an extensive analysis).

In this paper, we introduce GID framework to analyze social network communities. In this work we address the community detection problem as a game-theoretic approach employing Information Diffusion Model. Also, we extend the GADM model introduced by Lahiri and Cebrian [7] to achieve a more accurate model for Information Diffusion in social networks by defining a mutation operator. In contrast to the most of the existing methods, the proposed method does not rely on the structural attributes of the underlying network. Additionally, there is no parameter setting in the proposed approach.

\section{PROPOSED FRAMEWORK}

Informative human-human social interactions motivated us to assign the community formation problem in social networks to a play of interactions between their constituents, i.e. people. In real life, it is necessary for everyone to make friendship with those who have something valuable to share with, since humans are all sociable.

In this study, based on [8], we consider the community detection problem as a game in a multiagent environment and ascribe each vertex of the underlying graph to an agent. These agents try to form communities based on their utilities by joining to communities whose members have useful information for them. To quantify the information exchanged between agents, we introduce a new Information Diffusion Model (EGADM) based on the formulation in [7]. Finally, the local Nash equilibrium of the game corresponds to the clustering of the network. Algorithm 1 shows the proposed method, namely GID.

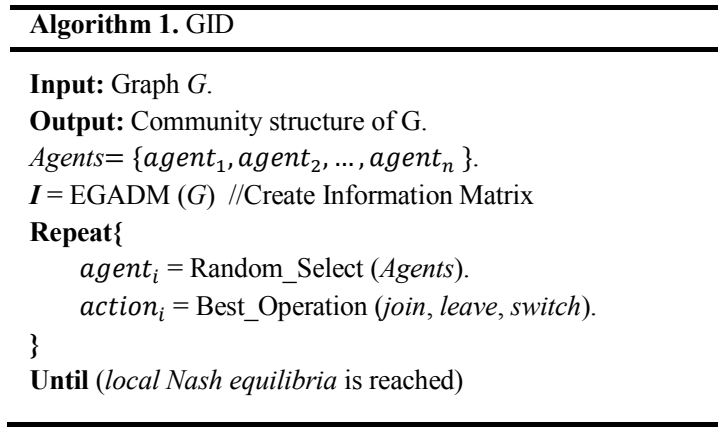

\section{EXPERIMENTAL RESULTS}

We now present our experimental results on three real world networks, a popular synthetic network and two benchmark social media datasets in [9]. We compare our algorithm results on synthetic networks with four other state- 
of-the-art algorithms in the area of community detection. We use two evaluation metrics to show the performance of our algorithm: Normalized Mutual Information (NMI) [10] and Modularity $Q$ [11]. The performance of all algorithms on three real-world networks in the form of Modularity and NMI is depicted in Table I.

TABLE I. The Average Results Of 100 Runs By GID, HA, MMC, LPA AND INFOMAP

\begin{tabular}{|c|c|c|c|c|c|c|}
\cline { 2 - 7 } \multicolumn{1}{c|}{} & \multicolumn{3}{c|}{ Q } & \multicolumn{3}{c|}{ NMI } \\
\cline { 2 - 7 } \multicolumn{1}{c|}{} & Dolphin & Karate & Football & Dolphin & Karate & Football \\
\hline GID & $\mathbf{0 . 5 3 8}$ & 0.373 & $\mathbf{0 . 5 9 8}$ & $\mathbf{0 . 7 1 5}$ & $\mathbf{1 . 0 0}$ & 0.838 \\
\hline HA [12] & 0.449 & $\mathbf{0 . 3 8 6}$ & 0.566 & 0.707 & 0.754 & 0.907 \\
\hline MMC [13] & 0.526 & 0.300 & 0.595 & 0.579 & 1.00 & 0.885 \\
\hline LPA [14] & 0.450 & 0.371 & 0.597 & 0.710 & 0.751 & $\mathbf{0 . 9 2 7}$ \\
\hline InfoMap [15] & 0.514 & 0.368 & 0.575 & 0.695 & 0.643 & 0.899 \\
\hline
\end{tabular}

The performances of different algorithms compared with our algorithm, applied on LFR network [16] are shown in Fig. 1. As it can be seen in this figure, GID performs better than almost all the other methods when $\mu \leq 0.7$ while LPA and HA reach zero at $\mu=0.65$. When $\mu>0.75$, nearly all of algorithms perform equally. Finally, we apply GID on Flickr $^{1}$ and BlogCatalog 2 datasets to detect communities of the underlying graph. As long as there is no ground truth results about the number and structure of the communities in these datasets, we only show the results of our algorithm in Table II.

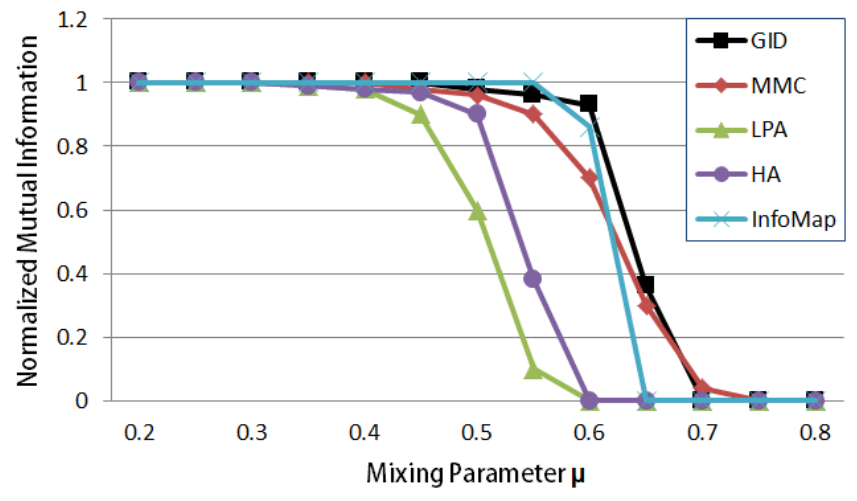

Fig. 1. Algorithms Performances on a LFR Synthetic Network (Communities with sizes of 20-100 on a graph with 10,000 nodes)

TABLE II. GID COMPUTATIONAL TIME AND NUMBER OF COMMUNITIES DETECTED ON BLOGCATALOG AND FLICKR

\begin{tabular}{|c|c|c|}
\hline Data Set & Flickr & BlogCatalog \\
\hline Time (Seconds) & 24,654 & 6,765 \\
\hline \# Communities & 132,765 & 15,645 \\
\hline
\end{tabular}

\section{CONCLUSION}

We have proposed a framework based on Game Theory and Information Diffusion Model to identify community structure of the underlying social network. In this study, we consider each node of the social network as a selfish agent which has interactions with its neighbors and tries to maximize its total utility (i.e. received information). Finally community structure of the graph reveals after reaching to the local Nash equilibrium of the game. The results demonstrate our method's superiority over other well-known methods.

\section{ACKNOWLEDGEMENT}

This work is supported by Iranian Telecommunication Research Center (ITRC) under Grant No. T/500/13226.

\section{REFERENCES}

[1] Flake G W, Lawrence S R, Giles C L, Coetzee F M., "SelfOrganization and Identification of Web Communities", IEEE Computer 35(3), 2002, 66-71.

[2] Girvan M, Newman M E J., "Community structure in social and biological networks". Proc. Natl. Acad. Sci. USA 99 (12), 2002, 7821-7826

[3] Chen J, Yuan B., "Detection of functional modules in the yeast protein-protein interaction network", Bioinformatics 22(18), 2006,2283.

[4] Newman M E J, Girvan M., "Finding and evaluating community structure in networks". Phys. Rev. E69 (2), 2004, 026113.

[5] Fortunato S., "Community detection in graphs", arXiv:0906.0612, 2009.

[6] J. Leskovec, K. Lang, and M. Mahoney, "Empirical comparison of algorithms for network community detection", in $W W W, 2010$ : Proceedings of the 19th international conference on World wide web, Apr 2010.

[7] M. Lahiri and M. Cebrian., "The genetic algorithm as a general diffusion model for social networks", Proc. of the 24th AAAI Conference on Artificial Intelligence (AAAI 2010), Atlanta, Georgia, 2010.

[8] Alvari, H., Hashemi, S., Hamzeh, A.: "Detecting Overlapping Communities in Social Networks by Game Theory and Equivalence Concept", AICI 2011, Part II, LNAI 7003, pp.620 - 630, 2011, Springer-Verlag.

[9] L. Tang and H. Liu., "Relational learning via latent social dimensions", In KDD '09: Proceedings of the 15th ACM SIGKDD international conference on Knowledge discovery and data mining, pages 817-826, New York, NY, USA, 2009. ACM

[10] L. Danon, A. Diaz-Guilera, J. Duch, and A. Arenas, "Comparing community structure identification", Journal of Statistical Mechanics:Theory and Experiment, vol. 9, p. 8, 2005.

[11] M.E.J. Newman and M. Girvan, "Finding and evaluating community structure in networks", Phys. Rev. E, vol. 69, no. 2, p. 026113, 2004.

[12] Newman M E J., "The structure and function of complex networks", SIAM Rev., 2003,45(2),167-263.

[13] M. Chen, "Discovering communities by Information Diffusion", Eighth International Conference on Fuzzy Systems and Knowledge Discovery (FSKD),2011.

[14] U.N. Raghavan, R. Albert, and S. Kumara, "Near linear time algorithm to detect community structures in large-scale networks", Phys. Rev. E, vol. 76, no. 3, p. 036106, 2007.

[15] M. Rosvall and C.T. Bergstrom, "Maps of random walks on complex networks reveal community structure", Proceedings of the National Academy of Sciences, vol. 105, no. 4, pp. 1118-1123, 2008.

[16] A. Lancichinetti and S. Fortunato, "Community detection algorithms: a comparative analysis", Phys. Rev. E, vol. 80, no. 5, p. 056117, 2009.

\footnotetext{
${ }^{1} \mathrm{http}: / / \mathrm{www}$. flickr.com

${ }^{2}$ http://www.blogcatalog.com
} 